\title{
MEASUREMENT OF SHORT-CIRCUIT EFFECTS ON TRANSFORMER WINDING WITH SFRA METHOD AND IMPACT TEST
}

\author{
Miroslav Gutten, Richard Janura, Milan Šebök, Daniel Korenčiak, Matej Kučera \\ University of Žilina, Faculty of Electrical Engineering, Univerzitná 1, 01026 Žilina, Slovakia \\ \iroslav.gutten@fel.uniza.sk,+421415132110,richard.janura@fel.uniza.sk,milan.sebok@fel.uniza.sk,daniel.korenciak@fel.uniza.sk, \\ matej.kucera@fel.uniza.sk)
}

\begin{abstract}
The paper presents theoretical and experimental analyses of a possible effect of the short-circuit forces on the transformer winding. The first part of the paper is focused on creation and activity of the radial and axial forces during a short circuit. It shows dimensions, direction and - of course - the resulting mechanical stress. The presented equation shows basic dependencies of these mechanical forces created in the transformer winding. Finally, the paper presents experimental methods of diagnosing and analysing the effects of short-circuit forces on the transformer winding.
\end{abstract}

Keywords: transformer, mechanical effects, measuring methods, frequency response, impact test.

(C) 2016 Polish Academy of Sciences. All rights reserved

\section{Introduction}

The worst mechanical effects acting on a transformer are caused by a sudden short circuit. Since the current flow through the windings in the case of a short circuit is enormous, the forces generated by it are also large and in many cases exceed the value of the maximum mechanical strength of windings or other parts of the transformer. Because of the transient and dynamic character of this phenomenon its solution is very difficult.

It is therefore necessary, in the absence of scientific and research potential in distribution and transmission organizations (e.g. power plants, heating plants), to achieve the objectives of the proposed activities, i.e. in-depth analysis of undesirable influence of short-circuit currents on the state of transformers, designing a modern methodology of measurements and its verification, and designing a new integrated diagnostic system enabling to analyse and classify the impact of possible failures caused by a short circuit on power transformers.

Apart from the winding failures (an inter-turn short circuit, a short-time connection with the tank) it could lead to destroying the tank because of the arc pressure. The direct cause of the forces acting on windings is the interaction of the magnetic field with current paths. In the case of a transformer it is the area of the leakage flux. In normal conditions, when the current values in the transformer do not reach the rated one, the forces acting on its windings are generally small. On the contrary, when they reach values which are multiples of the rated one, these forces could be dangerous for the windings and fixing construction [1].

The forces are created due to the interaction of the current and the magnetic flux density vectors. Thus, in general, the force vector can have any direction [2]. These forces can be radial (transverse) and axial (lengthwise) ones, therefore these two components of the force can be calculated and analysed independently [3]. Also, the two components influence different parts of the whole transformer and it is necessary to obtain the two components for design purposes. The terms: "axial" and "radial" apply to a concentric wound core type transformer. 
Action of the forces on the windings can be considered as a superposition of the separated radial and axial forces (Fig. 1). Both of these forces exist not only in the short circuit state but also in the rated state.

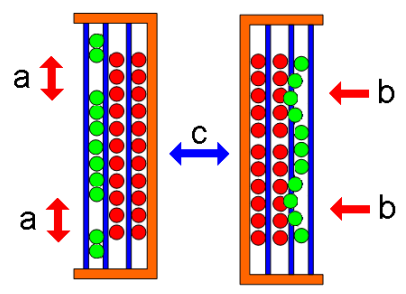

Fig. 1. The forces acting during a short circuit on the winding of power transformer ( $\mathrm{a}$ - axial effects, $\mathrm{b}$ - radial effects, $\mathrm{c}$ - rated state).

\section{Fundamentals of radial and axial forces}

The radial forces are those that act in the radial direction and are generated by interaction of the current and the axial component of leakage flux density. They tend to squeeze the inner winding and burst the outer winding. Calculation of radial forces does not present much difficulty since the axial component of leakage flux density is calculable fairly accurately [4].

Calculation of stresses due to this force acting on the conductors is more complex, especially for the inner windings. The compressive strength of winding is influenced by the radial thickness of conductors, its work hardening strength, the number of blocks per circle and the winding dimensions. Similarly, the outer winding experiences the outward radial force which results in the tensile stress. The tensile strength of winding depends on the work hardening strength of the conductors.

The radial forces are a result of the longitudinal field - parallel to the axis of the transformer winding. These forces stretch the outdoor windings and compress the internal winding, thereby increasing the air space between windings. The sum of the radial forces acts on the additional space between the windings - the outer primary winding has been moved to the outside space. The axial forces are those that act in the axial direction and are generated by interaction of the current and the radial component of leakage flux density. These forces are a result of flux created by the short-circuited conductor.

These forces can deform the windings in the lengthwise direction, and their overall addition operates on the coil of clamping ring and other clamping elements. Calculation of these forces causes big problems since the radial element of leakage flux density is difficult to compute exactly. When ampere turns are precisely aligned, so that the leakage flux pattern is symmetrical, then the leakage area is axial over the major part of the coil height. But, due to the flux lines dispersing in the radial direction in the vicinity of the winding ends, the axial flux density tends to decrease, and the resultant flux density at the ends can be resolved into the radial component causing the axial forces. These axial forces are unequally distributed between the outer and inner windings, due to the presence of core.

The axial forces at the top and bottom are in opposite directions as the currents are in the same direction. In the case the ampere turns are perfectly balanced and the leakage flux pattern is symmetrical, the resultant force acting on the winding would be zero. Any axial displacement between the magnetic centres of $\mathrm{HV}$ and LV windings will result in forces, which will increase the displacement $[5,6]$. 


\section{Experimental methods of diagnostics}

The biggest problem of today's energy transmission and distribution works is that the measured data from the diagnostic measurements are not adequately studied, since there is no scientific and research base. Therefore, assessing the technical condition of equipment, for example regarding its residual lifetime, is a major problem in deciding on the early decommissioning. At an unpredictable moment a failure may occur, and its consequence may be a long-time power failure. Even when using expensive commercial measuring devices, it is necessary to deeply analyse the measured data in relation to knowledge of exposure of the specific electrical equipment to adverse effects . Thus, achieving the objectives of this project may help to clarify the adverse effects and propose new diagnostic procedures that can be used to detect a forthcoming failure of the device, and to suggest certain steps in advance (e.g. an early substitution of a new device) [7].

With regard to the effects of short-circuit currents, over-currents and other phenomena which damage the winding and magnetic circuit, the following measurements will be performed on disconnected transformers:

- measurement of frequency characteristics by the SFRA method (Sweep Frequency Response Analysis);

- measurement of the time response of windings by a high voltage pulse source (the impact test);

- measurement of parameters of windings in the short-circuited state;

- a combination of diagnostic methods according to the proposed measurement procedures.

The use of different diagnostic methods and measurement procedures enables to make also measurements of connected transformers. These are thermography measurements, and monitoring of changes in electromagnetic radiation of transformers and their influence on measurement of interference with other devices, such as telecommunication or data devices.

When failures occur, e.g. as a result of a shift or inter-turn short-circuit in the winding of a transformer, the electromagnetic interference with other electrical apparatuses may change.

That is why the following measurements of connected transformers will be performed with an experimental technique:

- thermography measurement;

- measurement regarding electromagnetic interference of distribution apparatuses;

- additional measurements, or their combination, according to the proposed measurement procedures.

As a result of these measurements of transformers we can detect the effects of short-circuit currents, over-currents and other phenomena, which damage both winding and magnetic circuit of the transformer, without its dismantling and subsequent examination of failures, which is time-demanding [8].

Measurement of the frequency characteristics by the SFRA method, measurement of the time response of windings by a high-voltage pulse source and measurement of parameters of windings in the short-circuited state belong to methods that diagnose transformers without dismantling them. During the measurements there is no need of interfering into the construction of measured machine, and they are performed on a disconnected transformer [9].

\section{Experimental analysis of distribution transformer}

On the basis of applied algorithms and procedures performed in the first phase of solution presented in the paper, there were determined individual diagnostic methods and measuring techniques for measurements of a distribution transformer $22 \mathrm{kV} / 0.4 \mathrm{kV}$ with a defect in the 
high voltage phase A (Fig. 2). Our task was to compare sensitivity of the two methods - the SFRA method and a high voltage pulse source - by the impact test.

The SFRA method and the time response method (impact test) enable to determine the time response or frequency response of characteristic quantities of transformers. Measurement of a response in the time domain means finding out the time reaction to a certain voltage pulse at the input of winding. Measurement of a response in the frequency domain means finding out the amplitude (and phase in degrees) of a response to the harmonic voltage with a variable frequency at the input of winding. Whereas the response found out in the time domain is a record of the voltage behaviour in time, the response found out in the frequency domain depends on the amplitude response to frequency [10].

It is highly desirable to maximize the ability of detecting even a slightest movement in the winding-core arrangement of a power transformer while employing the SFRA test [11].

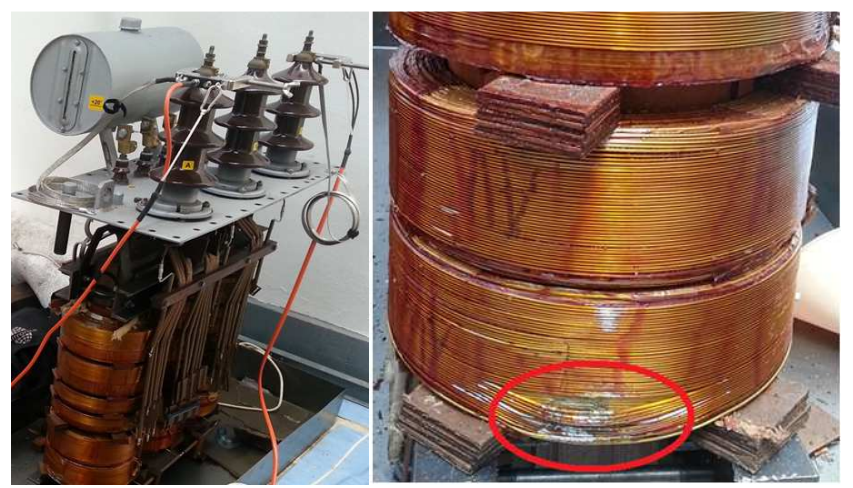

Fig. 2. A distribution transformer $22 / 0.4 \mathrm{kV}$ under the test in HV laboratory with a defect in the A phase.

\subsection{Analysis of transformer by SFRA method}

In measurement of a distribution transformer the frequency scale is set to at least $20 \mathrm{~Hz}$ to $2 \mathrm{MHz}$ at an output impedance $50 \Omega$ and a source voltage $0.2-20 \mathrm{Vpp}$, using Megger FRAX 150 (Fig. 3). The measurements were performed in the no-load and short-circuited states, according to the established standards.

During the no-load test, the mechanical state of tested winding and ferromagnetic core of transformer is detected. The measured curves provide the most important information about changes in the transformer core, which are visible in low frequencies and higher frequencies. They reflect either problems in winding movements or a turn-to-turn fault related to the shortcircuit forces [12].

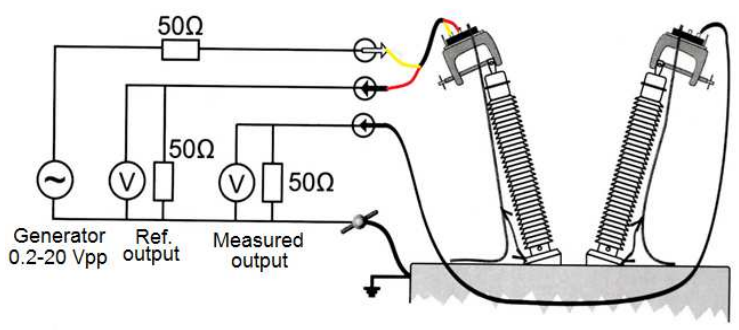

Fig. 3. SFRA test connection using FRAX 150. 
Differences in behaviour at low frequencies - up to about $1 \mathrm{kHz}$ - indicate problems due to movements of the transformer core, because of developing its resistance component. Differences in behaviour at middle frequencies - up to about $100 \mathrm{kHz}$ - indicate problems due to deformation and damage of transformer windings, because of developing the inductive component of coils. At high frequencies - more than $100 \mathrm{kHz}$ - problems due to damage of winding insulation or the contact resistance of transformer taps appear, because of developing the capacitance component.

During the short-circuit measurement mainly the winding state in primary or secondary parts of transformer is detected. These tests reliably indicate a deformation of the inner winding and its movement as a consequence of the short-circuit forces.

Analysis of the phase curve depending on frequency is suitable for more complex evaluation of the winding state. This analysis enables to assess the processes of winding deformation during the particular short-circuit effects.

Figure 4 shows the curves obtained from measurement of the impedance attenuation $(\mathrm{dB})$ in relation to frequency in the no-load and short-circuited states of the connection of A-C primary phases of the measured transformer. Further on, Fig. 5 displays the curves obtained from measurement of the phase (in degrees) in relation to frequency, using the same connection setup as mentioned for Fig. 4.

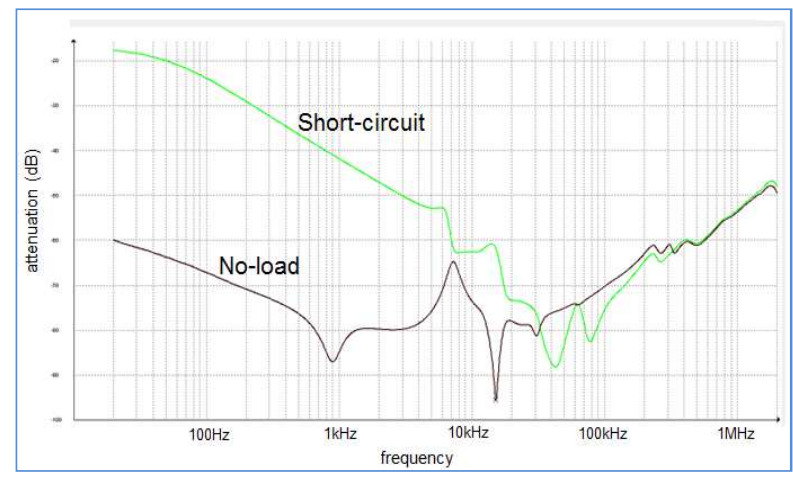

Fig. 4. Measurement of the impedance attenuation.

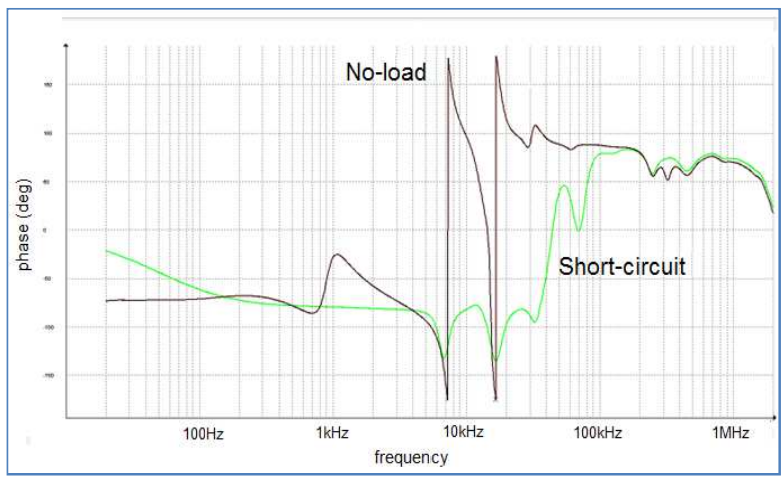

Fig. 5. Measurement of the phase in degrees. 
Comparing the above graphs, a clear difference is noticeable in the curves obtained from measurement of phase in relation to frequency (Fig. 5). It increases the analysis accuracy when defining the condition of the transformer.

Similarly, a significant difference is shown in Fig. 6, where an inter-threads' deformation of the coil $\mathrm{A}$ is most sensitively displayed by measurement of the phase in relation to frequency (about 1-10 kHz, see Fig. 6). It should be noted that, if the transformer windings are star connected, differences in the curves between phases $\mathrm{B}$ and $\mathrm{C}$ indicate a damage of another (i.e., A) phase coil.

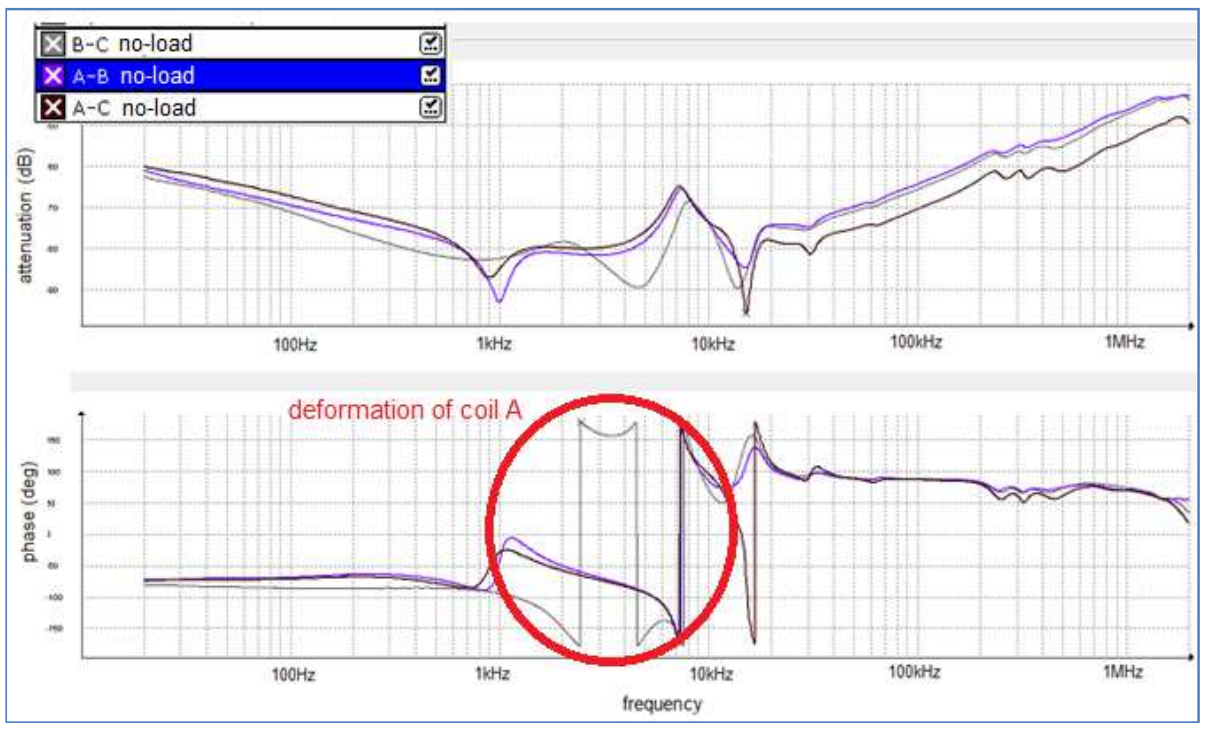

Fig. 6. Comparing the measurement results of attenuation and phase depending on frequency in the no-load test.

In order to analyse the measurement results it is possible to use the normalized mathematical standard "DL/T 911-2004". This calculation method mathematically analyses differences between two curves (sequences) using a relative factor $R_{\mathrm{xy}}$ in (1), (2) and (3).

Calculation of the standard variance of two sequences:

$$
D_{x}=\sum_{K=0}^{N-1}\left[X(k)-\frac{1}{N} \sum_{K=0}^{N-1} X(k)\right]^{2} \quad D_{Y}=\frac{1}{N} \sum_{K=0}^{N-1}\left[Y(k)-\frac{1}{N} \sum_{K=0}^{N-1} Y(k)\right]^{2},
$$

Calculation of the covariance of two sequences:

$$
C_{X Y}=\frac{1}{N} \sum_{K=0}^{N-1}\left[X(k)-\frac{1}{N} \sum_{K=0}^{N-1} X(k)\right]^{2} \times\left[Y(k)-\frac{1}{N} \sum_{K=0}^{N-1} Y(k)\right]^{2},
$$

Calculation of the normalization factor covariance of two sequences:

$$
L R_{X Y}=\frac{C_{X Y}}{\sqrt{D_{X} D_{Y}}} .
$$

Finally, from (9), the relative factor $R_{\mathrm{xy}}$ is determined [13].

The result of analysis in the no-load conditions according to (1) to (3) is the value of relative factor $R_{\mathrm{xy}}=0.35$ in the frequency range from $0.9 \mathrm{kHz}$ to $20 \mathrm{kHz}$ (Fig. 7), where the value of inductive part prevails, which results from the winding geometry. 
The above findings reveal a deformation anomaly due to an inter-threads' short-circuit, meaning a damage of coil A and thus threating the transformer operation (Fig. 2 - the visual defect).

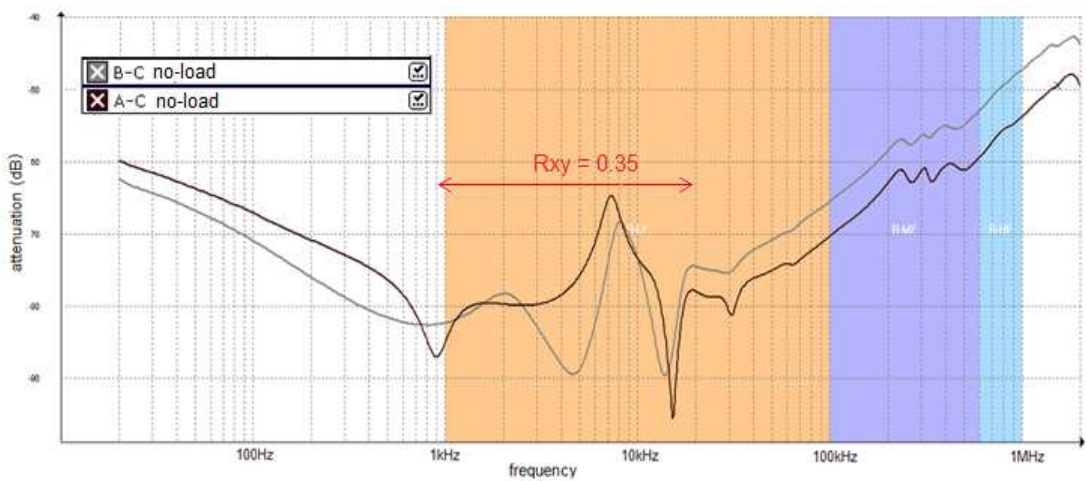

Fig. 7. The frequency analysis of deformation of coil A between the winding-phases A-C and B-C.

\subsection{Analysis of transformer by impact test}

The impact test (Fig. 8) is commonly used for testing the insulation among threads of coils or windings themselves and for searching the attenuated winding sections of electrical machines. This test enables to detect early stages of the winding faults. Short-time voltage pulses are applied to the coil in order to create a voltage gradient across the whole wire of the winding. This gradient produces an immediate voltage stress among the threads.

When the interference level is high enough, it might not be possible to distinguish the signal parameters from the recorded data. Conventional filtering methods cannot be useful for some interference, like white noise. In that case, the time-frequency filtering methods might be necessary [14].

In the time intervals between pulses the coil responds with damping oscillations of a sinusoidal shape. The unique nature of each coil response can be analysed by an oscilloscope. The wave shape is affected by a transient circuit dependent on the coil inductance and the internal capacity of the pulse generator. When an inter-thread occurs, the inductance decreases, thereby increasing the frequency of the response signal [15].

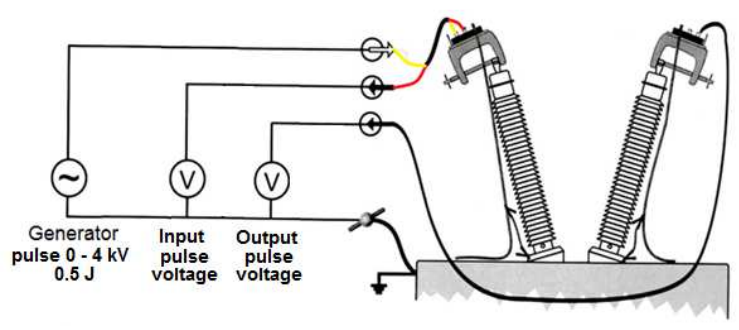

Fig. 8. Connection of a transformer in the impact test.

Figure 9 shows a comparison of the time-domain curves obtained from the pulse test measurement of a distribution transformer, where it is possible to observe a decreased time period from $9 \mathrm{~ms}$ to $12 \mathrm{~ms}$ caused by a defect of the coil. A potential amplitude decrease is caused by the change of the circuit resistance and capacitance due to a damaged conductor and 
winding insulation. The comparison is carried out on the windings of two phases, where phase $\mathrm{A}$ is affected by an inter-thread short circuit and phase B is fault-free.

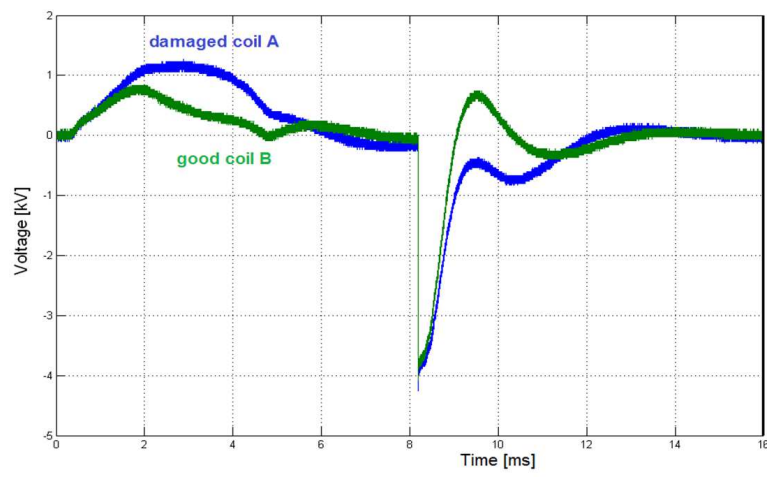

Fig. 9. Analysis of transformer coils with the impact test.

\section{Conclusion}

This paper emphasises the importance of knowing simulation and theoretical analysis of axial and radial forces, which cause irreversible deformation of transformer windings. The above analysis also shows the need to know the values of short-circuit current as it is critical and represents a significant danger to the transformer.

To apply the short-circuit current to a real transformer is not acceptable. In practice, we can diagnose a transformer by the frequency response method or the time response of windings by a high voltage pulse source. Both methods, SFRA and IM, indicate a significant sensitivity to a relatively small inter-thread deformation. These methods enable to directly and clearly analyse the short circuit impact on the transformer windings.

Even though the relation between the response curve and the condition of winding is clear, the overall subject is more complex. Based on the measured differences in the response, it is not possible to define a specific damage of the windings, but it is definitely possible to state that the condition of winding has changed, most likely deteriorated. Implementation of the mentioned methods in practice enables the user to monitor the transformer's state and decide on either its early check or maintenance (could be used as a preventive maintenance tool).

In order to obtain the frequency response curve, a procedure exists for recalculating the time response of the pulse measurement in the frequency domain. However, without IFRA equipment, the calculation is demanding and requires an oscilloscope with a large sampling frequency and memory. Information and outcomes mentioned in the paper are the basis for future research, which will focus on enlarging the knowledge of and determining clear relation among the frequency domain, the time domain and the condition of transformer windings.

\section{Acknowledgment}

This work was supported by project ITMS: 26210120021, co-funded from EU sources and European Regional Development Fund.

\section{References}

[1] Glowacz, A., Glowacz, A., Glowacz, Z. (2015). Recognition of Thermal Images of Direct Current Motor with Application of Area Perimeter Vector and Bayes Classifier. Measurement Science Review, 15(3), 119-126. 
[2] Gutten, M. (2011). Analysis of short-circuit currents in electrical equipment. Zilina: EDIS, 103.

[3] Koltunowicz, T.N., Zukowski, P., Boiko, O., Saad, A., Fedotova, J.A., Fedotov, A.K., Larkin, A.V., Kasiuk, J. (2015). AC Hopping Conductance in Nanocomposite Films with Ferromagnetic Alloy Nanoparticles in a PbZrTiO3 Matrix. Journal of Electronic Materials, 44(7), 2260-2268.

[4] Sedlák, J., Brandt, M., Seewald, R. (2012). Influence of Remanent Magnetization on the Diagnostic of Distribution 25MVA Transformer by SFRA Method. Elektro 2012 Proc., 465-468.

[5] Bharat Heavy Electricals Limited Bhopal. Transformers. (2003). New Dehli: McGraw Hill Education Private Limited, 614.

[6] Heatcote, M.J. (2007). The J \& P Transformer Book. Chennai: Elsevier, 989.

[7] Shayegani, A.A., Hassan, O., Borsi, H., Gockenbach, E., Mosheni, H. (2004). PDC Measurement Evaluation on Oil-Pressboard Samples. International Conference on Solid Dielectrics, 4, 50-62.

[8] Jurcik, J., Gutten, M., Korenciak, D. (2011). Analysis of transient actions influence in power transformer. Advances in Electrical and Electronic Engineering, 9(2), 65-69.

[9] Werelius, P., Öhlen, M., Adeen, L., Brynjebo, E. (2008). Measurement Considerations using SFRA for Condition Assessment of Power Transformers. International Conference on Condition Monitoring and Diagnosis, Beijing, China.

[10] Kvasnička, V., Procházka, R., Velek, J. (2005). Verification of method frequency characteristics in control room of distribution system Czech Republic. Diagnostika, Pilsen, Czech Republic.

[11] Arumugam, S. (2014). Experimental investigation on terminal connection and system function pair during SFRA testing on three phase transformers. International Journal of Electrical Power \& Energy Systems, 58, $101-110$

[12] Gutten, M., Jurčík, J., Korenčiak, D., Janura, R. (2014). Analysis of transformer winding considering transient effects. Journal of Electrical and Control Engineering (JECE), 4(3).

[13] Megger: Manual of instrument FRAX 150.

[14] Dikun, J., Onal E. (2015). Coherence and time-frequency analysis of impulse voltage and current measurements. Journal of Energy in Southern Africa, 26(2), 118-122.

[15] Ballon Instrument: Manual of generator PSG 204 A.

[16] Pavlanin, R., Dobrucky, B., Spanik, P. (2009). Investigation of Compensation Effect of Shunt Active Power Filter Working under the Non-Sinusoidal Voltage Conditions. International Review of Electrical Engineering - IREE, 4(5), 785-791. 